\title{
Editorial
}

Herz 2016 · 41:95-96 DOI 10.1007/s00059-016-4409-5

Online publiziert: 25. Februar 2016

๑) Springer Medizin Verlag 2016

\author{
R. Dörr ${ }^{1} \cdot$ R. Erbel ${ }^{2}$ \\ ${ }^{1}$ Praxisklinik Herz und Gefäße, Dresden, Deutschland \\ ${ }^{2}$ Institut für Medizinische Informatik, Biometrie, Epidemiologie, Universitätsklinikum Essen (AöR), \\ Essen, Deutschland
}

\section{Verbreitung medizintechnischer Innovationen in der klinischen Kardiologie}

\section{Ist Deutschland zu schnell oder zu langsam?}

Anlässlich der Herbsttagung der Deutschen Gesellschaft für Kardiologie Herz- und Kreislaufforschung (DGK) im Oktober 2015 in Berlin wurde vom amtierenden Präsidenten der DGK und Mitherausgeber dieser Zeitschrift, Prof. Dr. med. Karl-Heinz Kuck, mit Datum vom 8. Oktober 2015 die DGKPressemitteilung 10/2015 mit dem Titel „Kardiologischer Fortschritt in Gefahr? Nutzenbewertung und StudienAuflagen als Innovationsbremsen" veröffentlicht [1]. Prof. Kuck stellt darin fest, dass die Entwicklung in der Kardiologie in den vergangenen Jahren eine Erfolgsgeschichte sei. Einen nicht zu unterschätzenden Anteil an diesen Fortschritten habe die Medizintechnik, so Prof. Kuck. Von Stents zur Erweiterung von verengten Herzkranzgefäßen über Schrittmacher und implantierbare Defibrillatoren bis hin zur kardialen Resynchronisationstherapie hätten auch zahlreiche Innovationen aus der Medizintechnik dazu beigetragen, die Sterblichkeit aufgrund von Herzerkrankungen zu senken. Als Beispiel für die Dimensionen dieser Entwicklung verweist Kuck auf den aktuellen Herzbericht: Allein im Jahr 2012 wurden in Deutschland insgesamt 106.840 Herzschrittmachereingriffe (Neuimplantationen, Aggregataustausche, Revisionen) und 47.037 Defibrillatoreingriffe durchgeführt [2]. Aufgrund der neuen gesetzlichen Rahmenbedingungen zur Nutzenbewertung von neuen Medizinprodukten und aufgrund von strikten Auflagen für die
Durchführung von klinischen Studien mit Medizinprodukten sieht Kuck nun allerdings zunehmend Hürden für technische Innovationen - nicht nur, aber auch in der Kardiologie. Das Inkrafttreten des neuen Versorgungsstärkungsgesetzes [3] mit seiner Nutzenbewertung für bestimmte Medizinprodukte solle zwar nach dem erklärten Willen des Gesetzgebers für eine gute medizinische Versorgung auf hohem Niveau für alle Patienten sorgen, könne letztlich aber dazu beitragen, dass herzkranke Menschen in Deutschland nicht von den neuesten technischen Entwicklungen profitieren können. Dies weil diese Innovationen aufgrund eines CE-Zeichens zwar an sich zugelassen seien und verwendet werden dürfen, die Kosten aber von den Krankenkassen nicht übernommen würden. Nach den neuen gesetzlichen Bestimmungen müssen Medizinprodukte der Klassen IIb und III - das sind z. B. Implantate oder Herzschrittmacher - einer Nutzenbewertung unterzogen werden. Im Rahmen einer solchen Bewertung muss anhand von wissenschaftlicher Evidenz nachgewiesen werden, dass das neue Gerät, im vorliegenden Fall z. B. Schrittmacheraggregate mit "leadless pacing“, im Vergleich zu den bisher eingesetzten Verfahren für den Patienten einen klaren Zusatznutzen bringt. Das klinge zwar, so Prof. Kuck, durchaus einleuchtend, doch im Detail könne sich diese Betrachtungsweise als regelrechter Innovationshemmer erweisen. Denn die Methoden von Pharmastudien ließen sich nicht so einfach auf die Medizintechnik übertragen, so Prof. Kuck. Das Standardverfahren bei der Testung von Medikamenten, um den Nutzen und die Wirksamkeit einer neuen Substanz zu belegen, sei die placebokontrollierte Studie. Doch wie solle eine Placebokontrolle für einen Schrittmacher oder für einen medikamentenbeschichteten Stent aussehen? Es sei ja schon aus ethischen Gründen schwer vorstellbar, dass bei einem belastenden und niemals ganz unriskanten Eingriff mit Absicht ein nicht funktionierendes Gerät eingesetzt würde. Die Vorstellung, bei Patienten, die gefährdet sind, am plötzlichen Herztod zu versterben, einen Herzschrittmacher oder Defibrillator gegen ein Placebogerät testen zu müssen, erscheint den Herausgebern dieses Heftes in der Tat so absurd wie die Testung eines Fallschirms gegen Placebo bei einem Flugzeugabsturz.

Auch eine doppelte Verblindung, wie sie in der Medikamentenprüfung Standard sei, könne bei Eingriffen wie einer Schrittmacherimplantation nicht umgesetzt werden, so der DGK-Präsident. Die Beispiele machen aus seiner Sicht klar, wie schwierig der Beleg für den geforderten Zusatznutzen zu erbringen sein wird.

In Gegensatz $\mathrm{zu}$ dieser Position wurde in Anbetracht der schnellen Verbreitung einiger medizintechnischer Neuentwicklungen auf dem deutschen Markt immer wieder das europäische Zulassungsverfahren für Medizingeräte kritisiert, bei dem es wie bei elektrischen Haushaltsgeräten schon ge- 
nügt, dass ein medizintechnisches Gerät ein $\mathrm{CE}$ (Conformité Européenne)Zeichen der Europäischen Union besitzt, das dessen initiale Sicherheit beim Patienten („safety“) attestiert, ohne dass seine langfristige klinische Wirksamkeit („efficacy“) vor seiner Zulassung in großen randomisierten Studien nachgewiesen worden sein musste [4]. Dies kann dazu führen, dass ein bereits klinisch etabliertes Therapieverfahren aufgrund neuer wissenschaftlicher Erkenntnisse nachträglich revidiert und in den Leitlinienempfehlungen im Evidenzgrad zurückgestuft werden muss. Ein aktuelles Beispiel für dieses Phänomen ist die koronare Thrombektomie beim ST-Strecken-Hebungs-Infarkt (STEMI). Analoge Beispiele gibt es aus der kardiologischen Pharmakotherapie, wie z. B. beim Ivabradin, das zunächst hoch bewertet wurde, dann aber im Evidenzgrad herabgesetzt werden musste.

Vor dem Hintergrund dieser kontroversen Diskussion fand am 08.10.2015 während der Herbsttagung der DGK in Berlin eine hochinteressante, von der Arbeitsgruppe Interventionelle Kardiologie (AGIK) der DGK organisierte Sitzung mit dem Titel „Great Debate“ statt. Die Pro- und Kontra-Themen dieser Sitzung waren nicht nur kardiologisch spannend, sondern von hoher gesundheitspolitischer Relevanz im Hinblick auf die zukünftige Verbreitung innovativer Methoden in der interventionellen Kardiologie und der klinischen Elektrophysiologie.

In der ersten Pro-und-Kontra-Debatte ging es um das Thema „Wir machen zu viele TAVI“. Die Pro-Position wurde von PD Dr. med. Navid Mader, Herzchirurg aus Köln, vertreten. Den KontraPart hatte Prof. Dr. med. Hüseyin Ince, Berlin, aus der Sicht des interventionellen Kardiologen übernommen. Das zweite kontroverse Thema lautete „Wir implantieren zu viele ICD“. Das Pro-Referat hielt Prof. Dr. med. Dirk Böcker, Hamm, und die Kontra-Position vertrat Prof. Dr. med. Michael Block, München.

Im dritten Teil der Pro-und-KontraDebatte ging es um das Thema „Wir sind zu schnell mit neuen Methoden“. Das Pro-Referat hielt Prof. Dr. med. Anno Diegeler, Herzchirurg aus Neustadt a. d.
Saale, das Kontra-Referat der Mitherausgeber dieses Themenheftes, Prof. Dr. med. Raimund Erbel aus Essen.

Da die Themen dieser "Great Debate“ von sehr großem allgemeinen Interesse waren (selbst Präsident Prof. Kuck musste in der Sitzung stehen), haben die Herausgeber noch am 08.10.2015 in Berlin den spontanen Entschluss gefasst, die Referate zu veröffentlichen, um sie einem noch breiteren Publikum vorzustellen. Erfreulicherweise haben alle Referenten sofort zugesagt, zu ihren Referaten ein Manuskript für die Zeitschrift Herz einzureichen, sodass wir Ihnen hiermit alle Pro- und Kontra-Positionen mit der jeweils umfassenden Argumentation anbieten könnten.

Die Herausgeber wünschen Ihren bei der Lektüre dieses aus unserer Sicht sehr spannenden Themenheftes mit dem Titel „Great Debate“ viel Vergnügen.

Dr. med. Rolf Dörr

Prof. Dr. med. Raimund Erbel

\section{Korrespondenzadresse}

\section{R. Dörr}

Praxisklinik Herz und Gefäße, Heinrich-Cotta-Str. 12, 01324 Dresden doerr@praxisklinik-dresden.de

\section{Literatur}

1. Kardiologischer Fortschritt in Gefahr? Nutzenbewertung und Studien-Auflagen als Innovationsbremsen, Pressetext 10/2015 der Deutschen Gesellschaft für Kardiologie - Herz- und Kreislaufforschung (DGK) vom 08.10.2015

2. Deutscher Herzbericht 2014, Deutsche Herzstiftung e.V. in Zusammenarbeit mit den Fachgesellschaften DGK, DGTHG und DGPK, 2014, ISBN 978-3-9811926-6-7

3. Gesetz zur Stärkung der Versorgung in der gesetzlichen Krankenversicherung (GKV-Versorgungsstärkungsgesetz - GKV-VSG) vom 16. Juli 2015, Bundesgesetzblatt Jahrgang 2015, Teil I Nr. 30, ausgegeben zu Bonn am 22. Juli 2015, S 12111244

4. Marktzugangsvoraussetzungen für Medizinprodukte - Zuständigkeiten in Deutschland, Stand: Juni 2010. Rechtliche Grundlagen europäisch. http://www.bmg.bund.de/fileadmin/ dateien/Downloads/M/Medizinprodukte/ Medizin_Produkte_Marktzugangsvoraussetzungen_fuer_Medizinprodukte.pdf

\section{Eine der Ursachen für ischämisch bedingtes Nierenversagen gefunden}

Eine der häufigsten Auslöser von akutem Nierenversagen ist eine Ischämie. Wissenschaftlern von Berliner Einrichtungen MDC, Charité, FMP und der medizinischen Hochschule Hannover haben nun eine der Ursachen für ischämisch bedingtes Nierenversagen gefunden. Mit bildgebenden Verfahren hat das Wissenschaftler-Team erstmals am lebenden Organismus gezeigt, dass das zelluläre Signalprotein NF-KB in der Niere nach einer Ischämie aktiviert wird. Dieser Transkriptionsfaktor bewirkt auch in zahlreichen anderen Gewebetypen das Ablesen von Genen, die unter anderem mit programmiertem Zelltod, Entzündungs- und Immunreaktionen in Verbindung stehen. Wegen seiner vielfältigen Aufgaben im Körper ist die ungezielte medikamentöse Hemmung des Proteins nicht erwünscht. Es wurde ein Mausmodell entwickelt, in dem das NF-KB ganz spezifisch in den TubulusEpithelzellen der Niere inaktiviert wurde. In diesen Mäusen sahen die Forscher weit weniger Gewebeschäden, Nekrosen und Entzündungsherde nach einer künstlich herbeigeführten Ischämie. Der Grund dafür ist die zurückgefahrene Aktivierung von NF-kB-Zielgenen in der Niere. In Kulturen von Tubuluszellen ließ das Unterdrücken des Signalwegs die Zahl der abgestorbenen Zellen und die Ausschüttung von Entzündungsfaktoren zurückgehen. Da sie den Wirkmechanismus von NF-KB auf einen Zelltyp festlegen konnten, hoffen die Wissenschaftler, so den Grundstein für die Erforschung zukünftiger, auf diese Zellen zugeschnittene Therapien gelegt zu haben. Das zellspezifische Mausmodell kann des Weiteren leicht auf andere Zielproteine angepasst werden und kann für die Untersuchung weiterer Nierenerkrankungen wertvoll sein.

Literatur: L. Markó, E. Vigolo, C. Hinze et al (2015) Tubular epithelial NF-KB activity regulates ischemic AKI. J Am Soc Nephrol DOI: 10.1681/ASN.2015070748 